Post-print version of:

Publisher: Wiley

Journal paper: International Journal for Numerical Methods in Biomedical Engineering, 32(6): e02747

Title: CT segmentation of dental shapes by anatomy-driven reformation imaging and B-spline modelling

Authors: S. Barone, A. Paoli, A.V. Razionale

Creative Commons Attribution Non-Commercial No Derivatives License

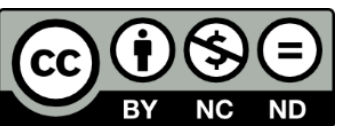

DOI Link: https://doi.org/10.1002/cnm.2747 


\title{
CT segmentation of dental shapes by anatomy-driven reformation imaging and B-spline modelling
}

\author{
S. Barone ${ }^{1}$, A. Paoli ${ }^{1}$ and A. V. Razionale ${ }^{1}$ \\ ${ }^{1}$ University of Pisa, Department of Civil and Industrial Engineering \\ Largo Lucio Lazzarino, 1 \\ 56126 Pisa - ITALY \\ $\S$ Corresponding author \\ Tel. $+39(050) 2218019$ \\ Fax +39 (050) 2218065 \\ email: a.paoli@ing.unipi.it \\ e-mail: s.barone@ing.unipi.it, a.paoli@ing.unipi.it, a.razionale@ing.unipi.it
}

\begin{abstract}
Dedicated imaging methods are among the most important tools of modern computer-aided medical applications. In the last few years, Cone Beam Computed Tomography (CBCT) has gained popularity in digital dentistry for 3D imaging of jawbones and teeth. However, the anatomy of a maxillofacial region complicates the assessment of tooth geometry and anatomical location when using standard orthogonal views of the CT data set. In particular, a tooth is defined by a sub-region, which cannot be easily separated from surrounding tissues by only considering pixel grey-intensity values. For this reason, an image enhancement is usually necessary in order to properly segment tooth geometries.

In this paper, an anatomy-driven methodology to reconstruct individual $3 \mathrm{D}$ tooth anatomies by processing CBCT data is presented. The main concept is to generate a small set of multi-planar reformation images along significant views for each target tooth, driven by the individual anatomical geometry of a specific patient. The reformation images greatly enhance the clearness of the target tooth contours. A set of meaningful 2D tooth contours is extracted and used to automatically model the overall $3 \mathrm{D}$ tooth shape through a B-spline representation.

The effectiveness of the methodology has been verified by comparing some anatomy-driven reconstructions of anterior and premolar teeth with those obtained by using standard tooth segmentation tools.
\end{abstract}

Keywords: Cone beam computed tomography, 3D imaging, Tooth segmentation, Digital dentistry, Bspline modelling 
Digital medical imaging plays an important role in modern clinical practice since it has enabled the development of computer-aided methods for both initial diagnosis and treatment planning. In dentistry, digital models of tooth geometries, including roots and crowns, are of utmost importance for simulating prosthetic assessments or predicting corrections of bite irregularities. The first requirement of a computer-aided approach is the identification of target tooth anatomies through a sequential imaging and reconstruction process.

Although, a large number of clinical assessments can only require 2D imaging by uni-planar x-ray transmissions and/or panoramic radiographies, a thorough treatment planning could take advantage of a 3D reconstruction from multi-planar images by Computed Tomography (CT) [1]. In the last few years, Cone Beam CT (CBCT) has gained popularity in dentistry for 3D imaging of jawbones and teeth. Unlike conventional Multi-Slice CT (MSCT) scanners, CBCT is suited for use in clinical dental practice where cost and radiation dose considerations are important, space is often at a premium and scanning requirements are limited to the head. The greatest practical advantage of CBCT relies on the ability to provide volumetric data sets through slice-wise inspections of standard orthogonal views. However, the reconstruction of dental tissues involves the inspection of hundreds of slices to evidence tooth regions, which cannot be easily separated from surrounding anatomies (i.e., bone tissues) by only considering pixel's grey-intensity values. The global thresholding of tooth roots could indeed lead to under or over segmentation problems due to the high similarity of grey intensity values between alveolar bone and dental structures. A key objective of medical image analyses is to improve the segmentation tasks for faster processing without sacrificing accuracy and completeness of the anatomy reconstructions.

Technical literature [2-9] has focused on adaptive thresholding techniques [2], active contour methods (i.e., snakes approach, level set approach), or hybrid methodologies, which combine different information into one energy functional for optimization.

Active contour methods, based on level set formulation, turned out to be the most promising approaches [3-6]. Level set methods present key features, which bring significant advantages for CT tooth segmentation: the contour curves can be either convex or concave; moreover, they can both expand and shrink, and naturally resolve topological changes simultaneously segmenting multiple objects [5].

Hybrid methodologies [7-9] have been also developed by exploiting information deriving from external scanned data (i.e., optical scanning of dental plaster models) to guide the segmentation of CT based medical images. The fusion of data deriving from different scanning technologies allows the reconstruction of high quality tooth models, even if these methodologies require patient's 
dentition plaster models.

The above-mentioned approaches rely on the slice-by-slice segmentation of the whole CBCT volumetric data set in the two-dimensional transverse plane. This requirement theoretically allows the achievement of exact tooth geometries, but the segmentation procedure should be supported by automatic techniques owing to the high number of involved slices. Moreover, the slice-by-slice approach cannot be considered intrinsically robust due to the nonhomogeneous intensity distribution inside each tooth and the low image contrast between the tooth root and the alveolar bone. For this reason, some enhancement would be advisable in order to properly segment overall tooth geometries. The present work is focused on the development of a procedure to reconstruct 3D shapes of individual dental anatomies by tracing only a small number of significant tooth contours. The underlying idea relies on restricting the tooth segmentation task to the analysis of a small set of plain images extracted from CBCT data through an anatomy-driven process [10]. The aim is at defining a robust approach to the problem, which is conceptually different from traditional slice-by-slice approaches.

The procedure starts with the creation of an augmented synthesized panoramic radiograph (a-PAN) by unwrapping the volumetric data set through the Discrete Radon Transform (DRT). The a-PAN is efficiently used to identify the bounding region including the tooth to be segmented (target tooth). A small set of local multi-planar reformation images is then automatically created in the form of Maximum Intensity Projection (MIP) images along the most significant projection directions, whose selection is guided by the knowledge of the specific anatomical morphology. The generation of local MIP images greatly enhances the clearness of the root contours, which can then be interactively outlined. A set of meaningful 2D tooth contours is obtained and used to automatically extract a Bspline curve for each axial slice, thus approximating the overall 3D tooth anatomy. The proposed approach allows the automatic creation of 2D images over significant and anatomy-driven planes, instead of using standard axial, coronal and sagittal planes, thus augmenting the visualization of the target tooth morphology.

The effectiveness of the methodology has been evaluated by experimental comparisons with standard segmentation tools.

\section{The anatomy-driven segmentation methodology}

A CBCT scan yields a stack of slices (Figure 1-a) corresponding to cross-sections through a maxillofacial volumetric region. CBCT data are stored in a sequence of Digital Imaging and Communications in Medicine (DICOM) images. An imaging slice is a 2D matrix of grey intensity values representing the $\mathrm{x}$-ray attenuation of different anatomical tissues. 
In this paper, the issues arising from tooth $\mathrm{CBCT}$ segmentations are addressed by creating a synthesized reference PAN image and four local Maximum Intensity Projection (MIP) images for each target tooth. These plain images are automatically defined on the basis of the specific anatomical morphology and are used to reconstruct single tooth shapes along with the relative spatial arrangements.

The overall methodology can be schematized as follows:

1) Medial axis identification

- identification of the medial axis of a dental arch by image processing techniques;

2) Augmented synthesized panoramic radiograph

- generation of an augmented synthesized panoramic image (a-PAN) obtained by superimposing two panoramic images created by unwrapping the CBCT volume through the Discrete Radon Transform (DRT);

3) Target tooth identification

- extraction of a volume around the individual target tooth by interactively outlining a profile onto the a-PAN;

- identification of the 3D tooth axis by exploiting four different projecting directions for the DRT;

\section{4) Creation of the MIP images}

- extraction of four reference planar sections perpendicular to the projecting directions;

- generation of four MIP images by $i$ ) thickening the reference planar sections, $i i$ ) extracting a set of slice images from the thickened volume, iii) assigning the highest pixel values of the slice set to the planar sections;

- outlining of 2D tooth contours on the MIP images;

\section{5) Tooth shape modelling}

- modelling a periodic B-spline curve for each slice perpendicular to the tooth axis and reconstruction of the $3 \mathrm{D}$ target tooth.

Each single stage of the methodology is fully detailed in the following sections.

\subsection{Medial axis identification}

An automatic procedure to determine the medial axis of the horseshoe shaped region of the dental arch has been developed. The steps of the procedure are summarized in Figure 1.

Preliminarily, the original DICOM slices are processed by a $3 \times 3$ Gaussian operator in order to reduce the background signal noise. A plain representation of the maxillofacial anatomy (maxillofacial 
image) is then obtained by displaying the maximum intensity value of the horizontal DICOM slices (Figure 1-a). The plain image is composed of the highest grey intensity values extracted from pixels having the same location in the DICOM stack images (Figure 1-b).

The plain maxillofacial image is binarized by using a threshold value determined by the Otsu's method [11] (Figure 1-c). The threshold value may undergo to slight variations in order to guarantee the achievement of a horseshoe-shaped region, which coarsely represents the plane description of the jaw anatomy (mandible or maxilla depending on the placement of the target tooth).

The binarized image is then subjected to a morphological closing by using a 5 pixels diameter circular-shaped structuring element. This operation preserves the circular nature of the object, at the same time filling little holes and connecting neighbouring structures (Figure 1-d). The algorithm proceeds with the labelling of all the connected regions and the automatic selection of the region presenting the greater area value (Figure 1-e). The selected region is skeletonised by a morphological thinning that successively erodes away pixels from the boundary, while preserving the end points of line segments, until no more thinning is possible (Figure 1-f).

A reference curve (medial axis) of the maxillofacial anatomy is determined by fitting the skeleton line with a second-order polynomial curve (dotted blue line in Figure 1-g). The medial axis approximates the dental arch morphology and represents an effective reference to synthesize panoramic radiographs by $\mathrm{CBCT}$ data.

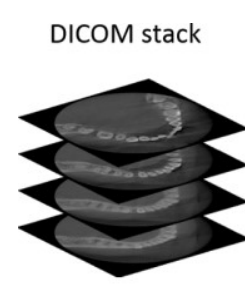

(a)

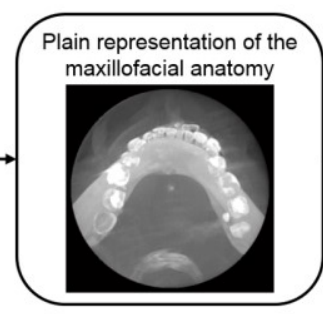

(b)

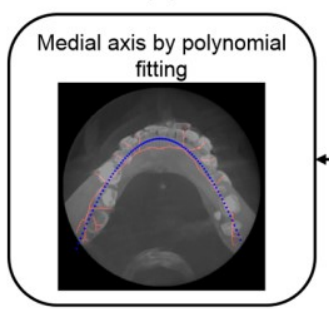

(g)

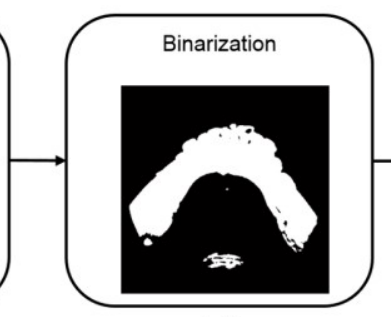

(c)

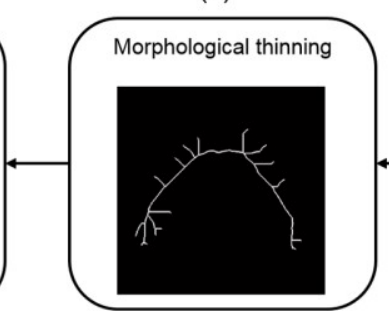

(f)

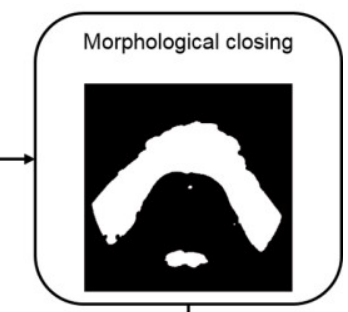

$(\mathrm{d})$

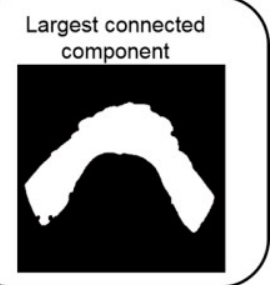

(e)

Figure 1 - Scheme of the procedure developed to identify the medial axis of the plain representation of a patient's jaw. DICOM stack images (a), generation of the maxillofacial image (b), binarization process (c), morphological closing (d), identification of the largest connected region (e), skeleton extraction by morphological thinning (f), medial axis computation (dotted blue line) by fitting the skeleton (superimposed with a continuous red line) (g).

\subsection{Augmented Synthesized Panoramic Radiograph}

A panoramic radiograph essentially represents the sum of $\mathrm{x}$-ray attenuation along each ray transmitted 
from the source to a film. The attenuation is due to the x-ray beam absorption and scattering by incident human tissues. A current research trend is focalised on the development of automatic or semi-automatic methodologies for synthesizing panoramic radiographs by unwrapping CT-scan volumetric data [12-16]. These synthesized panoramic radiographs are often used in clinical treatments since they exhibit reduced blurring, geometric distortion and overlapping of other dental structures. Moreover, they contain an amount of information, which is not readily available within tomographic data sets. In particular, PAN images describe the entire mouth, which is a threedimensional curved structure, in a single plain image, including the teeth, upper and lower jaws, surrounding tissues.

In this paper, a first PAN radiograph is synthesized from the CBCT data set by summing the grey intensity values of voxels disposed along projection lines normal to the reference curve as obtained in the previous section. The process is accomplished for each slice by performing the DRT, which consists of taking a line integral along a line (ray), which passes through the object space. In particular, finite pixel sums are computed by using the DRT for a restricted region contained between two offset curves obtained from the reference curve (Figure 2-a).

The first synthesized PAN image (Figure 2-b) is augmented by superimposing a second PAN image obtained through the DRT projection of the 3D digital model of the jaw and dentition. Practically, a three-dimensional triangular mesh of the isosurface within the volumetric CBCT data set at a specified grey intensity value (isovalue) is reconstructed. The raw 3D model of the maxillofacial region is then sliced along the vertical $\mathrm{z}$ direction with the same vertical resolution of the volumetric data set (Figure 3-a). For each slice $\mathrm{S}$, the intersection $(\Gamma)$ between the slice plane and the 3D model is computed and a 2D binary image $\left(I_{S}\right)$ (Figure 3-b) is created accordingly to:

$$
I_{S}(x, y)=\left\{\begin{array}{cc}
1 & \text { if } \quad(x, y) \in \Gamma \\
0 & \text { otherwise }
\end{array}\right.
$$

The second panoramic image (Figure $3-c)$ is then obtained by unwrapping the $\left(I_{S}\right)$ binary image data set through the DRT along the projection lines normal to the same reference curve used to unwrap the CBCT data set.

The two synthesized panoramic images are superimposed in order to obtain an augmented representation (a-PAN) of the maxillofacial region (Figure 3-d). 


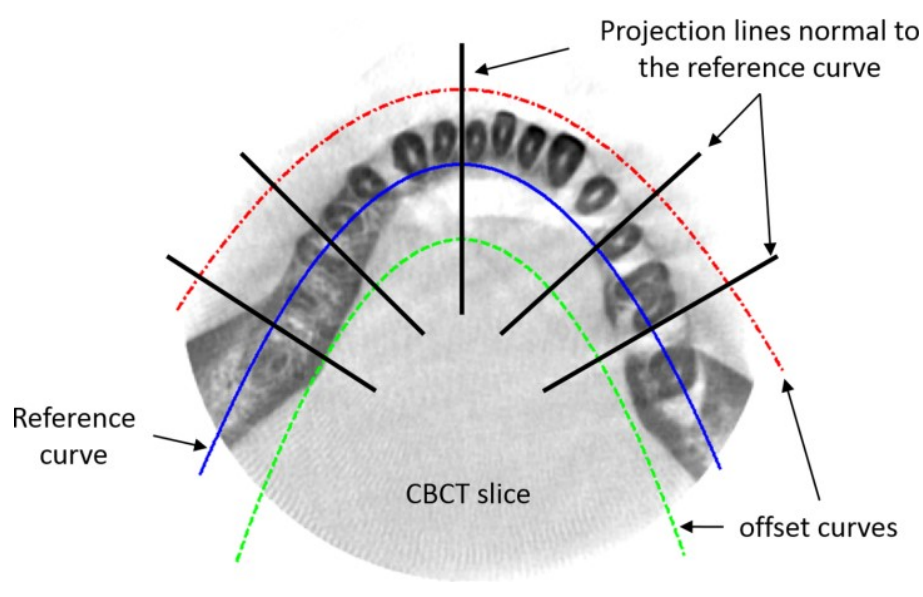

(a)

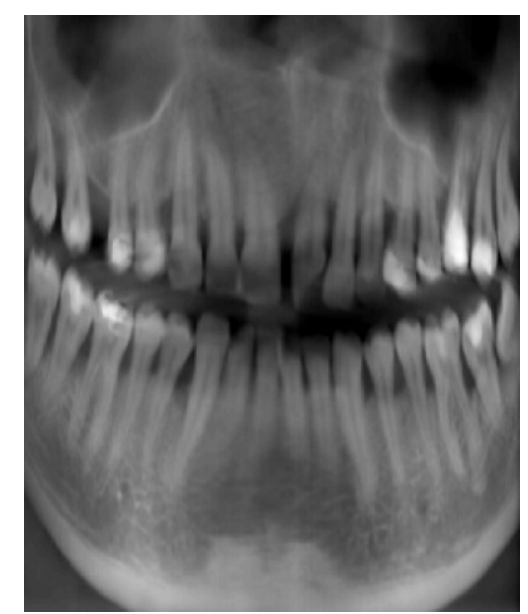

(b)

Figure 2 -Projection lines normal to the reference curve and projected region delimited by the offset curves (a) and synthesized panoramic radiograph (PAN) (b).

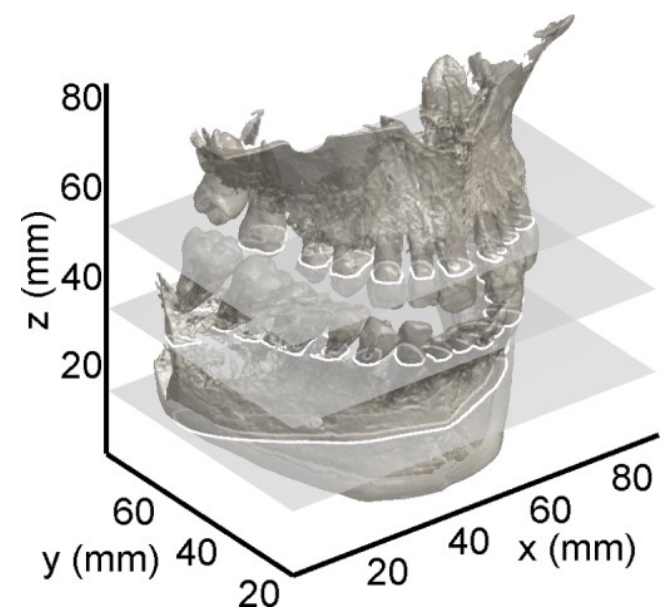

(a)

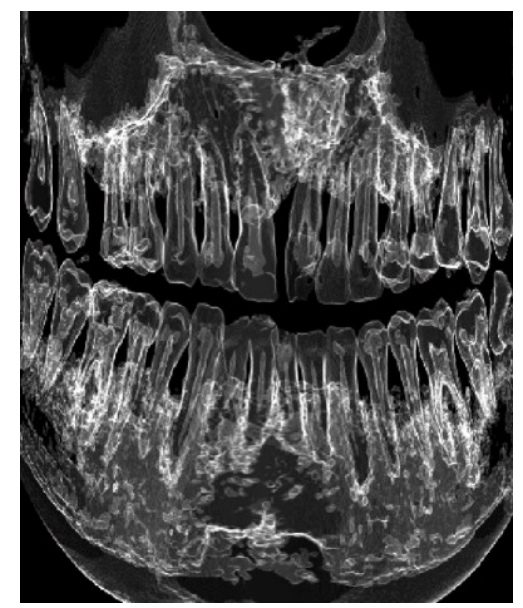

(c)

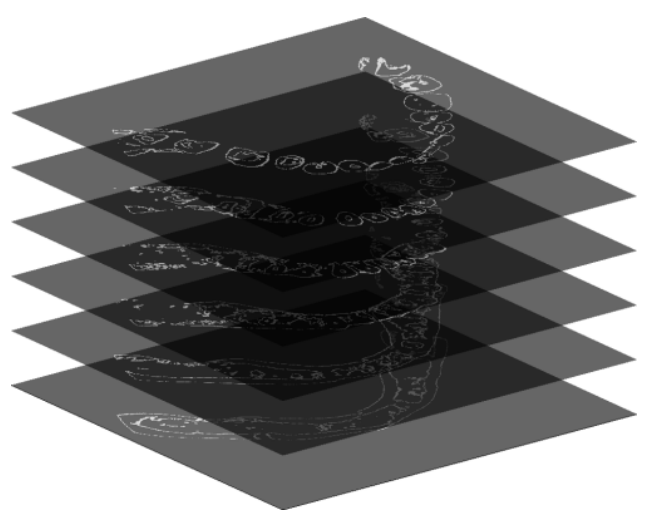

(b)

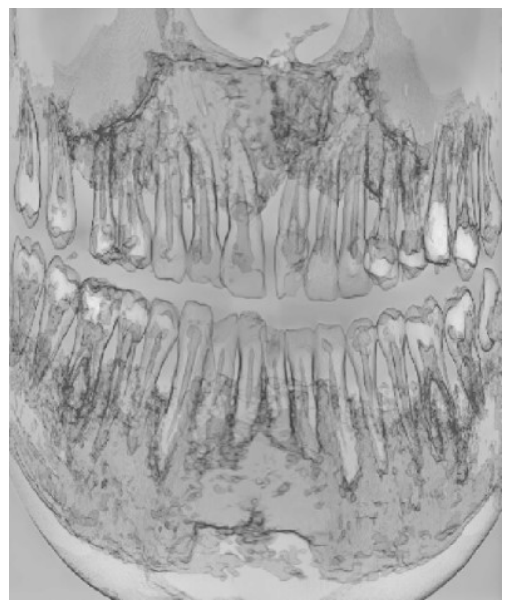

(d)

Figure 3 - Slicing the 3D model (a), binary images data set (b), synthesized panoramic image (c) obtained by performing the DRT to the binary data set, augmented representation (a-PAN) (d) obtained by superimposing the two synthesized panoramic images. 


\subsection{Target tooth identification}

The a-PAN is exploited to outline a rough 2D contour around a target tooth (Figure 4-a). The 2D contour is used for locating a bounding volume of the target tooth within the whole CBCT data, thus restricting the identification of the reference data for the generation of the anatomy-driven MIP images. The two outermost points of the contour are automatically determined in each slice of the DICOM stack (i.e., points A and B in Figure 4-a). The slice image is partitioned into four distinct regions defined by two lines, which are perpendicular to the medial axis and pass through the outermost points (Figure 4-b). Among these regions, the area containing the target tooth is automatically filled (Figure 4-c) and intersected with the largest connected region in the binarized maxillofacial image (Figure 1-e). The intersection operator provides a narrower segmented area, which certainly contains the target tooth region in the processed slice (Figure 4-d). The whole stack of segmented areas creates a bounding volume, which encloses the overall target tooth shape (Figure 4-e). This procedure restricts the search domain of the target tooth in the following segmentation steps.

The next process consists in the identification of the tooth axis by computing the DRT projections of the bounding volume by exploiting four significant different directions (viewing directions). In particular, direction 1 corresponds to the bisector of the angle between the two lines, which are perpendicular to the medial axis and pass through the outermost points (Figure 4-b), traced in the middle slice within the bounding volume. Directions 2, 3 and 4 are orientated at +45 degrees, 90 degree and - 45 degrees with reference to direction 1, respectively (Figure 5-a). Directions 1 and 3 correspond to meaningful clinical views, since direction 1 represents the buccolingual view and direction 3 represents the mesiodistal view. Figures from 5-b to 5-e show the projection images of the bounding volume along the four significant directions. These images are used to manually identify the $2 \mathrm{D}$ tooth axes as evidenced by the superimposed continuous line segments. The $3 \mathrm{D}$ tooth axis is finally obtained by reconstructing the four directions of projection passing from the bottom of the tooth (dotted lines in Figure 6-a) and the four directions of projections passing from the tip of the tooth (continuous lines in Figure 6-a). The two mean points obtained by averaging the mutual intersection points between bottom projection's directions and tip projection's directions are used to identify the 3D tooth axis (arrowed continuous black line in Figure 6-a).

The tooth volume is then re-oriented in order to dispose the normalized tooth axis vector, $\mathbf{r} \equiv\left(r_{x}, r_{y}\right.$, $r_{z}$ ), parallel to the normalized $\mathbf{z}$-axis vector (Figure 6-b) through a three-dimensional rotation around the centre of the tooth $C \equiv\left(c_{x}, c_{y}, c_{z}\right)$ defined as:

$$
T R=T_{f} \cdot R \cdot T_{b}
$$


where:

$$
\begin{gathered}
T_{f}=\left[\begin{array}{cccc}
1 & 0 & 0 & 0 \\
0 & 1 & 0 & 0 \\
0 & 0 & 1 & 0 \\
-c_{x} & -c_{y} & -c_{z} & 1
\end{array}\right], T_{b}=\left[\begin{array}{cccc}
1 & 0 & 0 & 0 \\
0 & 1 & 0 & 0 \\
0 & 0 & 1 & 0 \\
c_{x} & c_{y} & c_{z} & 1
\end{array}\right], \\
R=\left[\begin{array}{cccc}
r_{x}^{2}(1-\cos \vartheta)+\cos \vartheta & r_{x} r_{y}(1-\cos \vartheta)-r_{z} \sin \vartheta & r_{x} r_{z}(1-\cos \vartheta)+r_{y} \sin \vartheta & 0 \\
r_{x} r_{y}(1-\cos \vartheta)+r_{z} \sin \vartheta & r_{y}^{2}(1-\cos \vartheta)+\cos \vartheta & r_{y} r_{z}(1-\cos \vartheta)-r_{x} \sin \vartheta & 0 \\
r_{x} r_{z}(1-\cos \vartheta)-r_{y} \sin \vartheta & r_{y} r_{z}(1-\cos \vartheta)+r_{x} \sin \vartheta & r_{z}^{2}(1-\cos \vartheta)+\cos \vartheta & 0 \\
0 & 0 & 0
\end{array}\right] \\
\vartheta=\arccos (\mathbf{r} \cdot \mathbf{z})
\end{gathered}
$$

This process allows the target tooth to be always viewed with its real dimensions in all the MIP images created for the down streaming tooth reconstruction, thus facilitating the contour outlining process as described in the next paragraph.

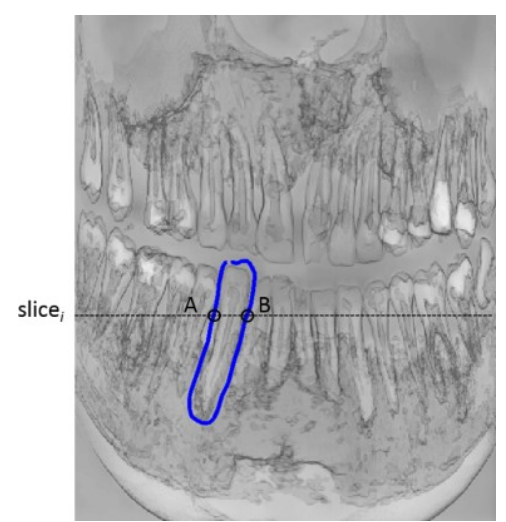

(a)

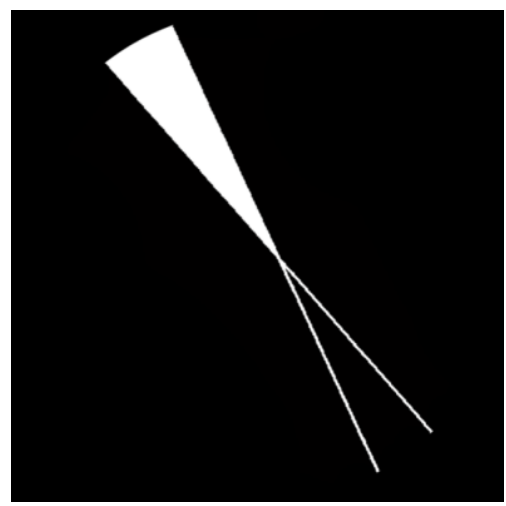

(c)

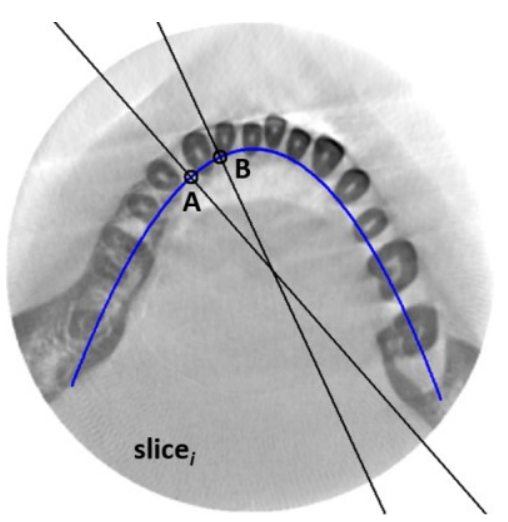

(b)

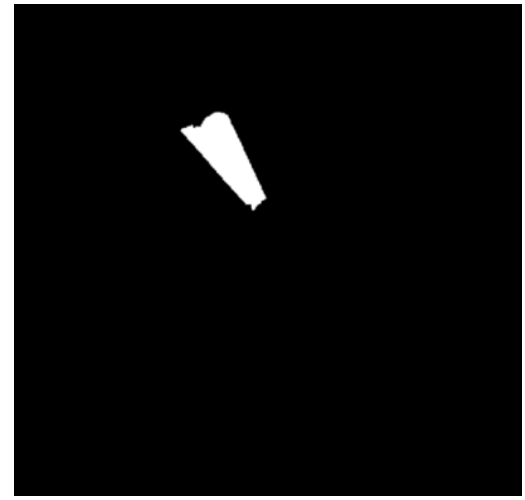

(d)

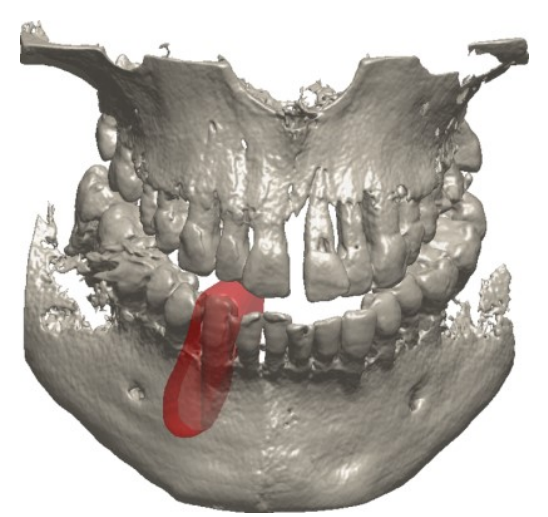

(e)

Figure 4-Outlining of the target tooth (a), partitioning of the slice area (b), flood-fill of the slice region containing the target tooth (c), narrower area containing the tooth contour (d), whole segmented 3D volume enclosing the overall tooth anatomy (e). 


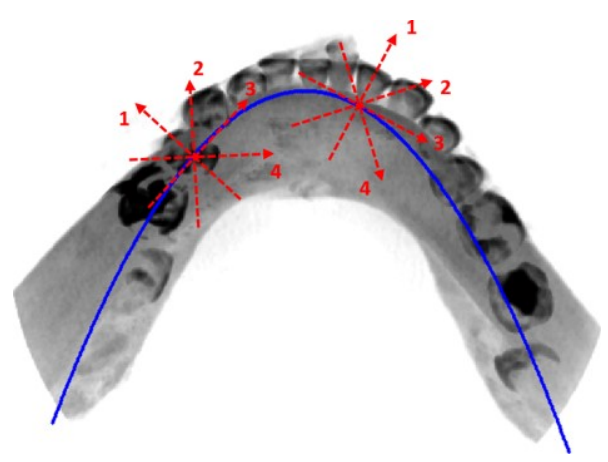

(a)

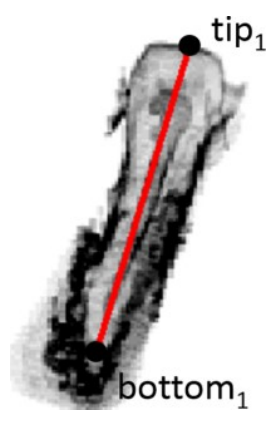

(b)

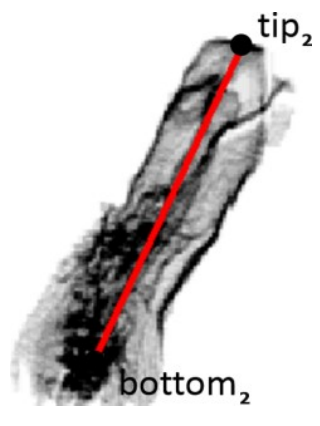

(c)

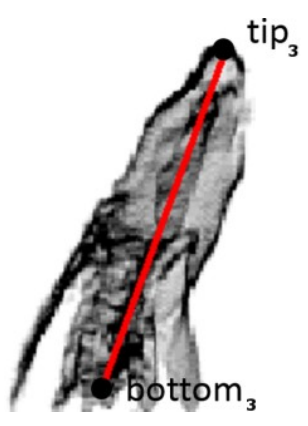

(d)

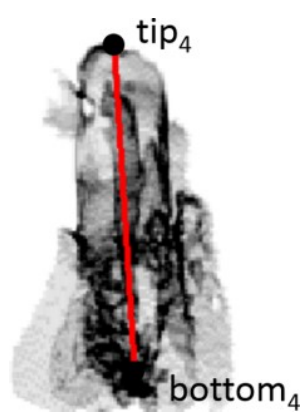

(e)

Figure 5 - Significant directions of two different target teeth used for the DRT projections (a), and projected results with the axes of a target tooth (b-e).

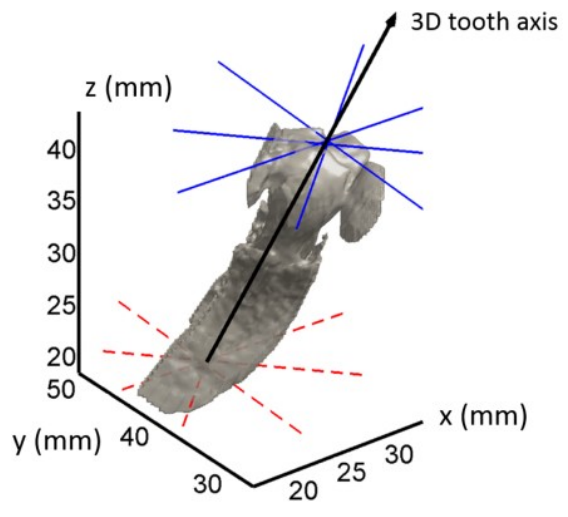

(a)

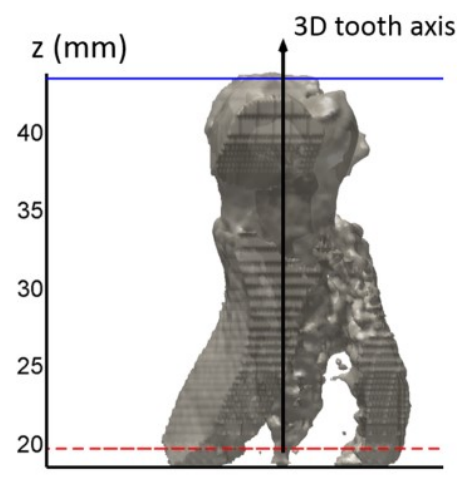

(b)

Figure 6 -Determination of the 3D tooth axis (a) and re-orientation of the whole target tooth volume (b).

\subsection{Creation of the MIP images}

Four reference planar sections are extracted as passing from the tooth axis and oriented perpendicular to directions 1, 2, 3, 4. Each reference planar section is "thickened" by considering a local neighbourhood (usually, within the range of \pm 8 voxels) along the respective viewing direction (Figure 7). A set of slice images is then extracted from the thickened volume and a local MIP image is created on the reference planar section (reformation image plane) by using the highest pixel values of the slice set. As example, Figure 8-a shows the four distinct MIP images obtained from the bounding volume of Figure 4-e. 
The thickening process fulfils two distinct roles. Firstly, it brings out the outermost (envelope) tooth contour when the tooth is viewed along the selected direction. The fulfilment of this requirement may not be accomplished by only considering the slice passing through the target tooth axis (Figure 7). The MIP image does not necessarily point out the real tooth contour in correspondence of the planar section, rather represents a "pseudo" 3D structure, which contains information about a local tooth volume. Moreover, the visualization of anatomical structures different from the target tooth is weakened, thus enhancing the contrast between the tooth root and the alveolar bone. Dental practitioners are then facilitated in the outlining of the four different tooth contours (Figure 8-b). Figure 9 shows the four MIP images along with the corresponding manually outlined tooth contours (MIP contours) as spatially oriented within the CBCT volume with respect to the medial axis.

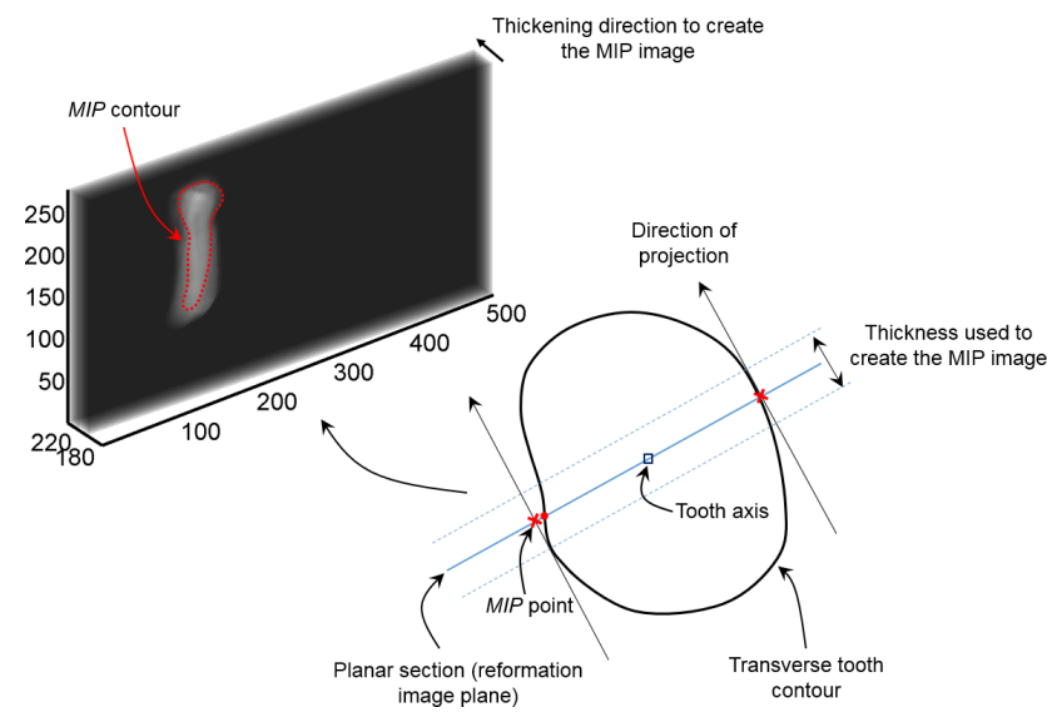

Figure 7 - Thickening process and extraction of a MIP image from the thickened volume.
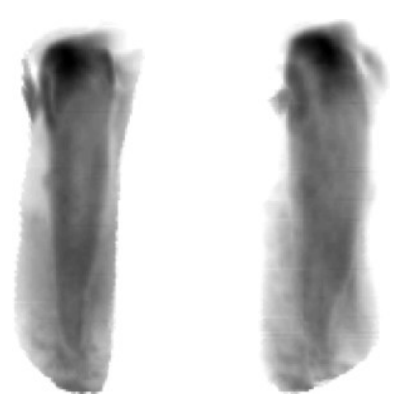

(a)
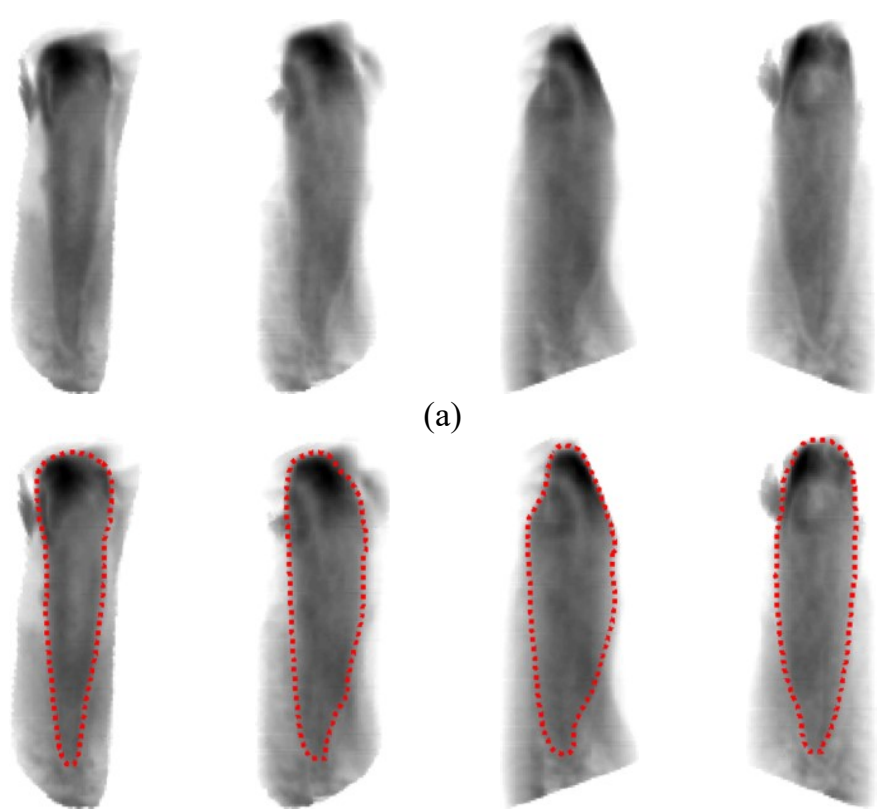

(b)

Figure 8-MIP images of the target tooth corresponding to the bounding volume of Figure 4-e (a) and manual outlining of the target tooth contours (b). 

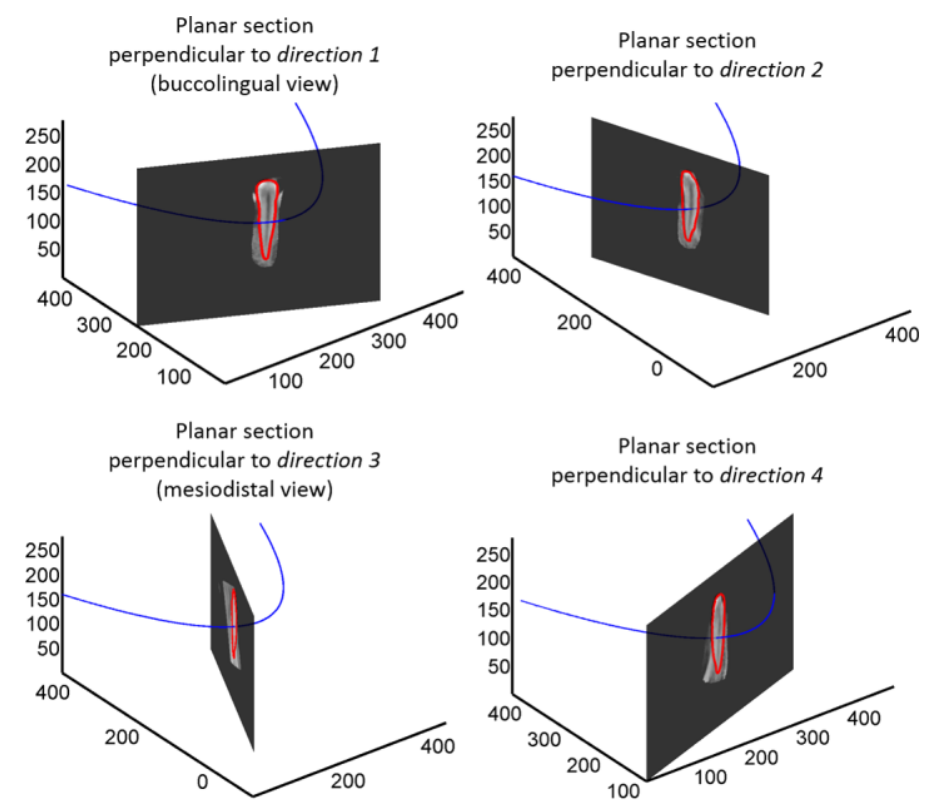

Figure 9-MIP images and tooth contours oriented within the CBCT volume with respect to the medial axis.

\subsection{Tooth shape modelling}

The aim of this procedure is to create a $3 \mathrm{D}$ representation of the individual dental shape by automatically contouring the transverse tooth sections belonging to the DICOM stack slices. Each slice intersects the MIP contours in eight points (MIP points) as shown by red marks in Figure 10-a. These points are not directly used for the contouring process since they are approximate representations of the target tooth. Indeed, the MIP contours are obtained by projecting actual points onto the respective planar sections as described in the previous paragraph. In this paper, a more accurate planar bounding area of the target tooth is defined by introducing a new set of points as intersections (blue circles in Figure 10-b) between eight lines passing through the MIP points and directed along the respective significant directions 1, 2, 3 and 4. Actually, a subset of these points is considered as control points for the successive B-spline modelling. In particular, the control points coincide with the vertices of the convex polygon obtained by twice discarding the convex polygons obtained through a convex hull algorithm: which is applied the first time to the intersection points, and the second time to the internal remaining points. Figure 10-c exhibits the first convex hull, with the outer green diamond marks, and the second convex hull, with the inner blue circle marks, which is obtained after the removal of the first convex hull (outer points). The remaining intersection points $\boldsymbol{P}_{\boldsymbol{i}}$, marked with yellow stars, describe a convex polygon, which certainly circumscribe the tooth contour. The set $\boldsymbol{P}_{\boldsymbol{i}}$ is used as control points for the creation of a periodic $B$-spline. It is advisable that the B-spline curve, which should model the tooth contour on the transverse slice, would be tangent to the significant directions. This is possible if intersection points of viewing direction, rather than 
MIP points, are used as control points of the B-spline curve. A parametric $B$-spline curve $C(u)$ of degree $p$ is defined by:

$$
\boldsymbol{C}(u)=\sum_{i=0}^{n} N_{i, p}(u) \boldsymbol{P}_{i}
$$

where $\boldsymbol{P}_{\boldsymbol{i}}(i=0, \ldots, n)$ are the $n+1$ control points and $N_{i, p}(u)$ are the normalized $B$-spline functions of degree $p$ defined on a knot vector $U=\left\{u_{0}, u_{1}, u_{2}, \ldots, u_{m}\right\}$, with $m=n+p+1$. In this paper, the periodic B-spline curve ( $p=2$ ) has been obtained by "wrapping" the control points $\boldsymbol{P}_{i}$. In particular, the last $p$ control points must be identical to the first $p$ control points $\left(P_{n}=P_{p-1}, P_{n-1}=P_{p-2}, P_{n-2}=P_{p-3}\right)$ and a uniformly spaced non-decreasing real-valued knot sequence of $m+1$ knots $\left(u_{0}=0, u_{1}=1 / m, u_{1}=2 / m\right.$, $\left.\ldots, u_{m}=1\right)$ is defined in the interval $[0,1]$. This formulation guarantees that the curve tangentially touches each line segment of the control polygon at its midpoint, thus following the shape outlined by the polygon. For each slice, 100 points are evaluated on the B-spline curve by using the de Boor's algorithm (Figure 10-d), in order to obtain a point cloud representing the overall tooth shape. Figure 11-a shows the three-dimensional coordinates of the control points as determined for each transverse slice. Figure 11-b and 11-c respectively show the resulting target tooth point cloud and the StL representation obtained by the point cloud tessellation.

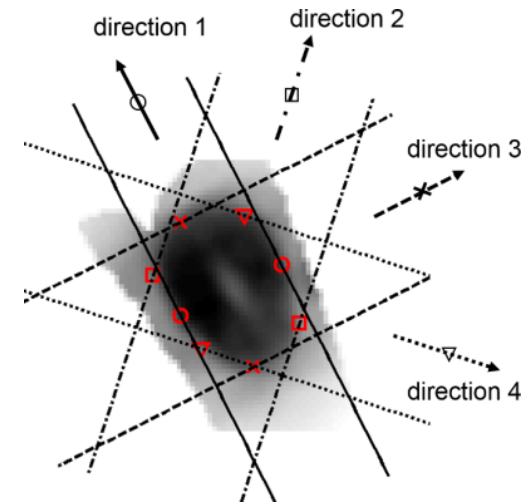

(a)

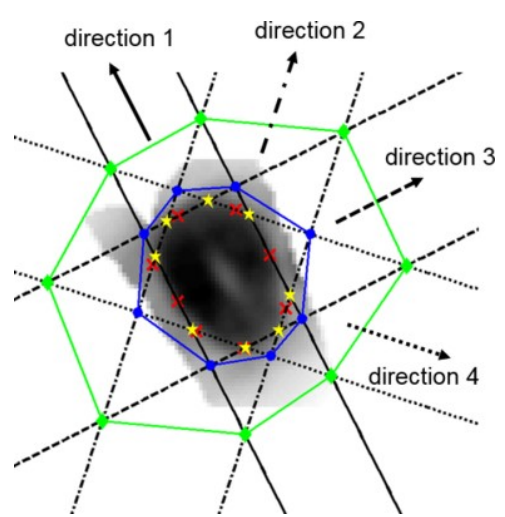

(c)

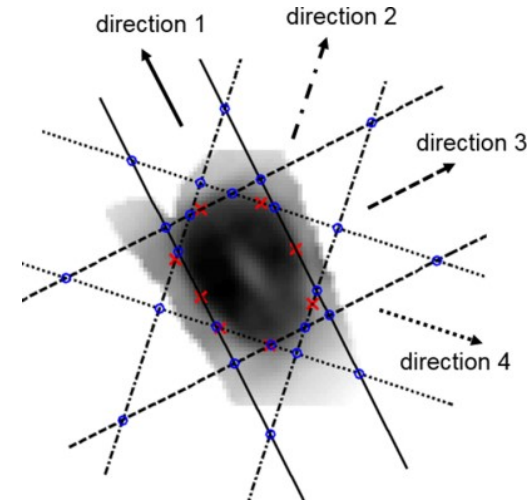

(b)

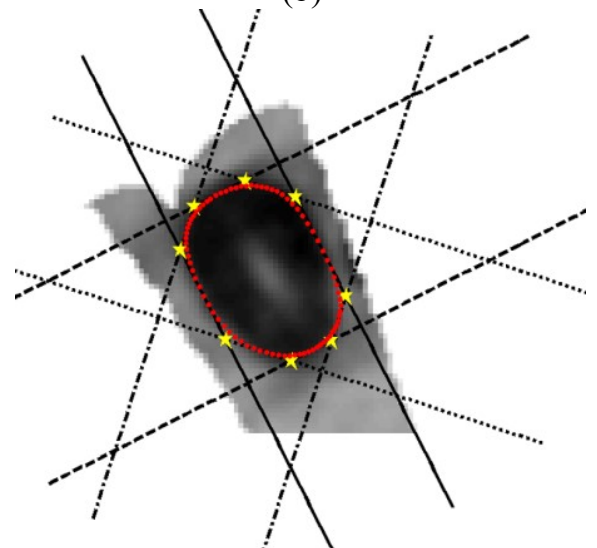

(d)

Figure 10 -Lines defined on a transverse slice by exploiting the four outlined tooth contours (a), intersection points (blue circles) (b), convex hull used to detect the polygon containing the tooth section (c), point sampling on the B-spline curve (d). 


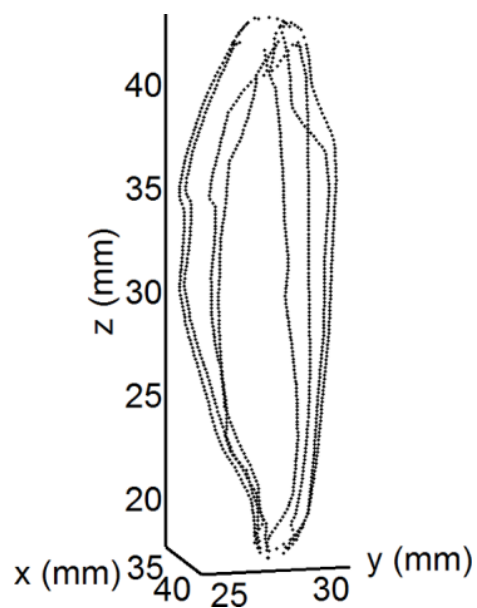

(a)

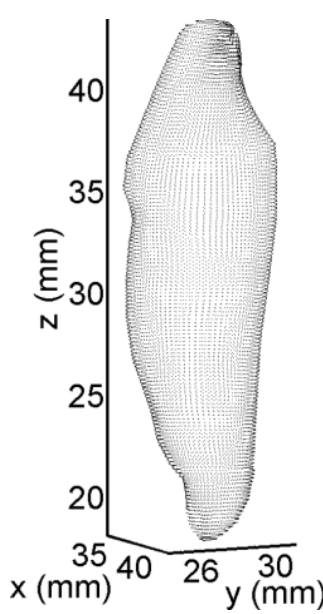

(b)

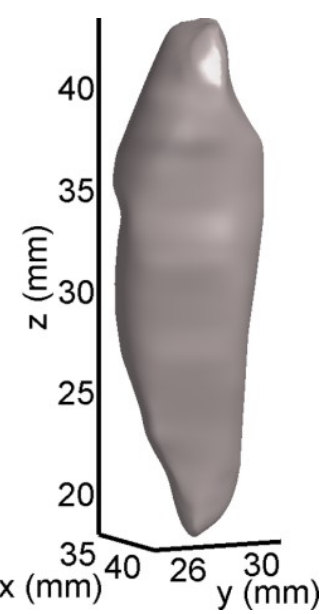

(c)

Figure 11 - Results of the segmented target tooth: 3D coordinates of the control points used for the middle points of the polygon line segments obtained for the whole slices stack (a), tooth point cloud (b), corresponding StL representation (c).

\section{$3 \quad$ Results and discussion}

The feasibility of the proposed approach has been assessed by analysing seven CBCT data sets describing oral and maxillofacial regions of different patients. The analysed data contain cases with prosthetic elements and metal artefacts due to dental fillings. Moreover, the data sets differ for both scanner manufacturers and spatial resolution. In particular, the spatial resolution varies from $0.18 \mathrm{~mm}$ $\times 0.18 \mathrm{~mm} \times 0.18 \mathrm{~mm}$ to $0.4 \mathrm{~mm} \times 0.4 \mathrm{~mm} \times 0.4 \mathrm{~mm}$, while the matrix resolution for each image slice varies from $250 \times 250$ pixel to $580 \times 580$ pixel (with the number of slices ranging from 200 to 450). The overall data set has allowed the reconstruction of 50 incisors, 25 canines and 50 premolars.

Figure 12 shows an example of two augmented synthesized panoramic radiographs as obtained by unwrapping two distinct CBCT data sets accordingly to section 2.2. The quality of these synthesized radiographs depends on the isovalue used to reconstruct the triangular meshes of the maxillamandibular region within the CBCT volume. However, this value does not affect the downstream tooth reconstruction process since the augmented synthesized panoramic radiograph is only used to isolate the target tooth by tracing a $2 \mathrm{D}$ contour around it. For this reason, the isovalue can be iteratively fine-tuned with the aim at enhancing the tooth contours in the synthesized radiograph.

A further consideration may regard the reliability of the procedure adopted to compute the reference curve used to unwrap CBCT data. Even if higher order polynomials or the use of B-spline curves, as documented in [13], could better approximate the dental arch, the results obtained have demonstrated that even a second order polynomial is effective in the reconstruction of a panoramic image functional to the target tooth selection. 
As example, Figure 13 reports the four MIP images obtained for an incisor, a canine and a premolar tooth as well as the respective 3D tooth anatomies (point cloud and StL representation) obtained as described in section 2.5.

The overall 3D tooth models are illustrated in Figures 14-a and 14-b for the patients described in Figure 12-a and 12-b, respectively. The segmented 3D tooth anatomies have been used to perform a quantitative validation of the proposed approach by comparing the reconstruction of anterior and premolar teeth with the results obtained by a manual slice-wise segmentation of standard orthogonal views. The manual segmentation has been performed by a clinician exploiting tools provided by open-source software for medical image analysis [17]. Figures 14-c and 14-d show the results obtained by using the reference tooth segmentation software for the patients described in Figures 12a and $12-b$, respectively.

The comparisons between Figures 14-a and 14-c and Figures 14-b and 14-d evidence how the proposed approach minimizes the staircase effect typical of surfaces obtained from transverse contours segmented slice-by-slice. The four contours traced on the MIP images guarantee a smooth transition between the transverse slices.

Figure 15 illustrates two different views of the full field discrepancies occurring by a best-fit alignment between the reconstructed shapes (Figure 14-a) and the reference models (Figure 14-b) relative to the patient represented in Figure 12-a.

Even if the analysis of the full field discrepancies on the overall dental arches gives a reasonable estimate of the tooth reconstruction accuracy, a more detailed investigation has been carried out. In particular, the mean $(\mu)$ and standard deviation $(\sigma)$ values of the discrepancies have been individually computed for each reconstructed tooth. Figures 16-a and 16-b report the graphical representations of these test metrics by using boxplot descriptions. Tooth numbers have been indicated in accordance with the ISO 3950 notation [18]. Teeth are symmetrically arranged within the mouth. Each quadrant hosts eight different teeth, which are horizontally and vertically mirrored with respect to the other quadrants. Each of these eight teeth is numbered from 1 to 8 , starting from the centre front tooth (central incisor) and moving backwards up to the third molar (number 8). Moreover, a number is assigned to each quadrant: from 1 to 4 for the adult (permanent) teeth. The combination of the quadrant number and the tooth number provides a two-digit code, which unambiguously identifies each tooth.

For each tooth, the Figures 16-a and 16-b report two distinct boxplots, which are differentiated by a colour gradient. The darker colour is used to represent the comparison between the results obtained by using the proposed approach and those composing the reference database. The lighter colour is 
used to depict the variability (precision) of the proposed approach. For each patient, the tooth segmentation process has been carried out by five different clinicians. Moreover, each boxplot reports the sample mean by drawing a continuous black circle.

The comparison with the reference data evidences that mean discrepancies are in the range $0.1 \div 0.3$ $\mathrm{mm}$, while standard deviations turned out to be included between $0.2 \mathrm{~mm}$ and $0.4 \mathrm{~mm}$, without any systematic difference between incisors, canine and premolar teeth. However, higher discrepancies have been observed for crowns geometries. Figure 17 shows the 2D comparisons between reconstructed teeth (dashed black line) and reference data (continuous red line) identified over the four reference planar sections. This circumstance was expected since crown contours are a combination of convex and concave curves, while root contours are generally straight or slightly convex, in particular for incisors and canine teeth.

Some considerations may be drawn in accordance to the evaluations performed by the clinicians after the segmentation of the presented cases. The possibility to have available a dedicated tooth visualization, driven by the individual anatomical geometry of the specific patient, has been considered a tool of utmost importance to facilitate the tooth contour outlining. The developed method simplifies the image segmentation task since it requires only four tooth contours to be outlined. The processing time is greatly reduced with respect to standard cumbersome slice-by-slice methods usually proposed within medical image software packages. An average time of three minutes has been recorded for the segmentation of each tooth by using the proposed method with respect to twenty minutes by adopting a slice-by-slice approach. A good approximation of the patient's teeth is indifferently obtained for incisors, canine and premolar teeth, in few minutes.

The analysis of the precision has been also carried out since the methodology requires some manual interventions. In particular, the outlining process of the tooth contours on the MIP images is manually performed, thus representing a possible error source. However, the criterion used to select the four viewing directions, which represent meaningful clinical views, and the generation of MIP images, enhances the clearness of the tooth contours, thus reducing the operator subjectivity in the outlining process. This results in a reasonable variability of the proposed approach as attested by the low spread $(<0.1 \mathrm{~mm})$ of the lighter colour boxes for the reconstructed teeth (Figure 16). The achieved accuracies, especially for what concerning crown geometries, cannot be considered acceptable to design prosthetic restorations and/or surgical implant templates. However, results can be considered adequate to plan orthodontic treatments. In clinical practice, the 3D modelling of root geometries of anterior and premolar teeth is a crucial diagnostic advantage when orthodontic treatments must be performed. This advantage is further leveraged for impacted teeth, as the case presenting an upper 
canine buried within the jawbone showed in Figure 12-b. The majority of aesthetic orthodontic treatments usually considers the movement of anterior teeth, and a good approximation of root geometries, as well as neighbouring structures, provides orthodontists with a clear 3D anatomical map to simulate trajectories and pathways during the treatment planning. In this context, the coarser reconstruction of crown geometries can be considered a minor issue. When orthodontic treatments require an accurate reconstruction of crown surfaces (i.e., those based on the use of customized removable appliances), the visible dental surfaces are usually reconstructed by using an intraoral scanner or by acquiring the plaster model with an optical scanner. This is due to the poor resolution of tomographic scanning, with respect to optical scanning, which causes inaccuracies in the crowns reconstruction thus leading to incorrect treatment simulations [7]. In these cases, the most accurate tooth reconstruction is obtained by merging data deriving from different sensors (optical scanning for crows and tomographic scanning for roots). The proposed tooth segmentation methodology is based on the convex hull property. For this reason, it is oriented to the reconstruction of anterior and premolar teeth, which usually present mono-radicular anatomies. If complex geometries have to be reconstructed (i.e., multi-radicular and/or multi-cusped teeth), more contours and more than one Bspline curve for each transverse slice should be used in order to manage changes in tooth topology when the shape split into different branches. This circumstance, however, has not been investigated in the present work.

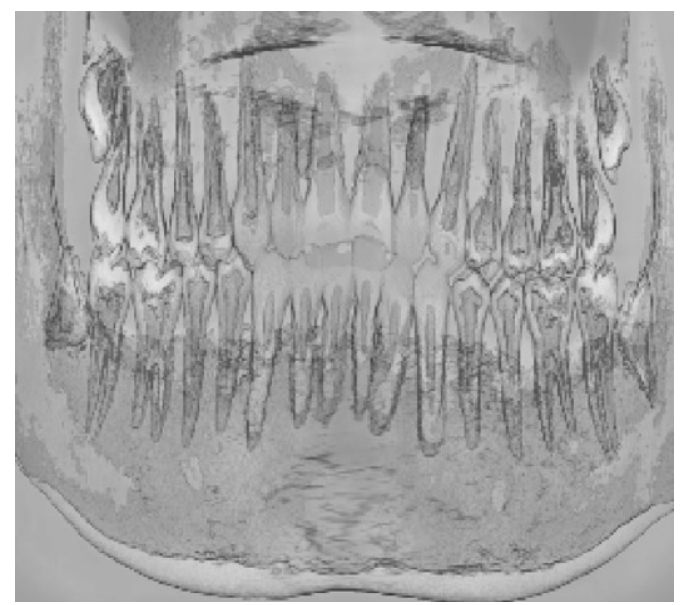

(a)

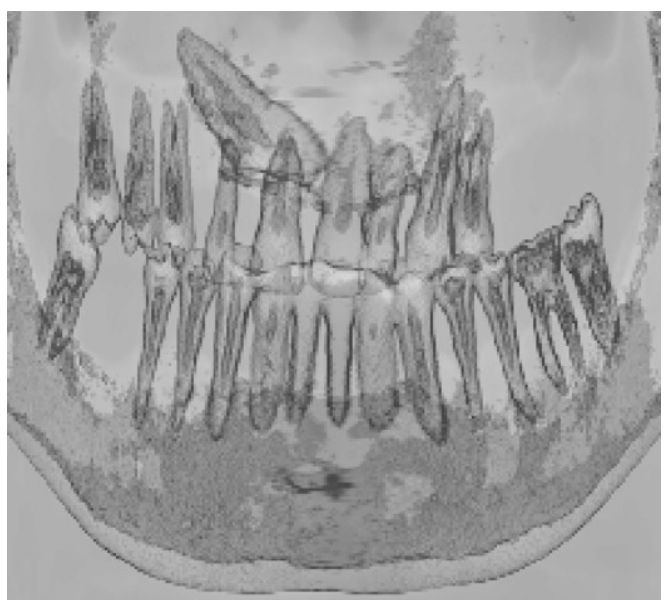

(b)

Figure 12 - Two examples of augmented synthesized panoramic radiographs (a-PAN) obtained by unwrapping CBCT data from two different patients. 

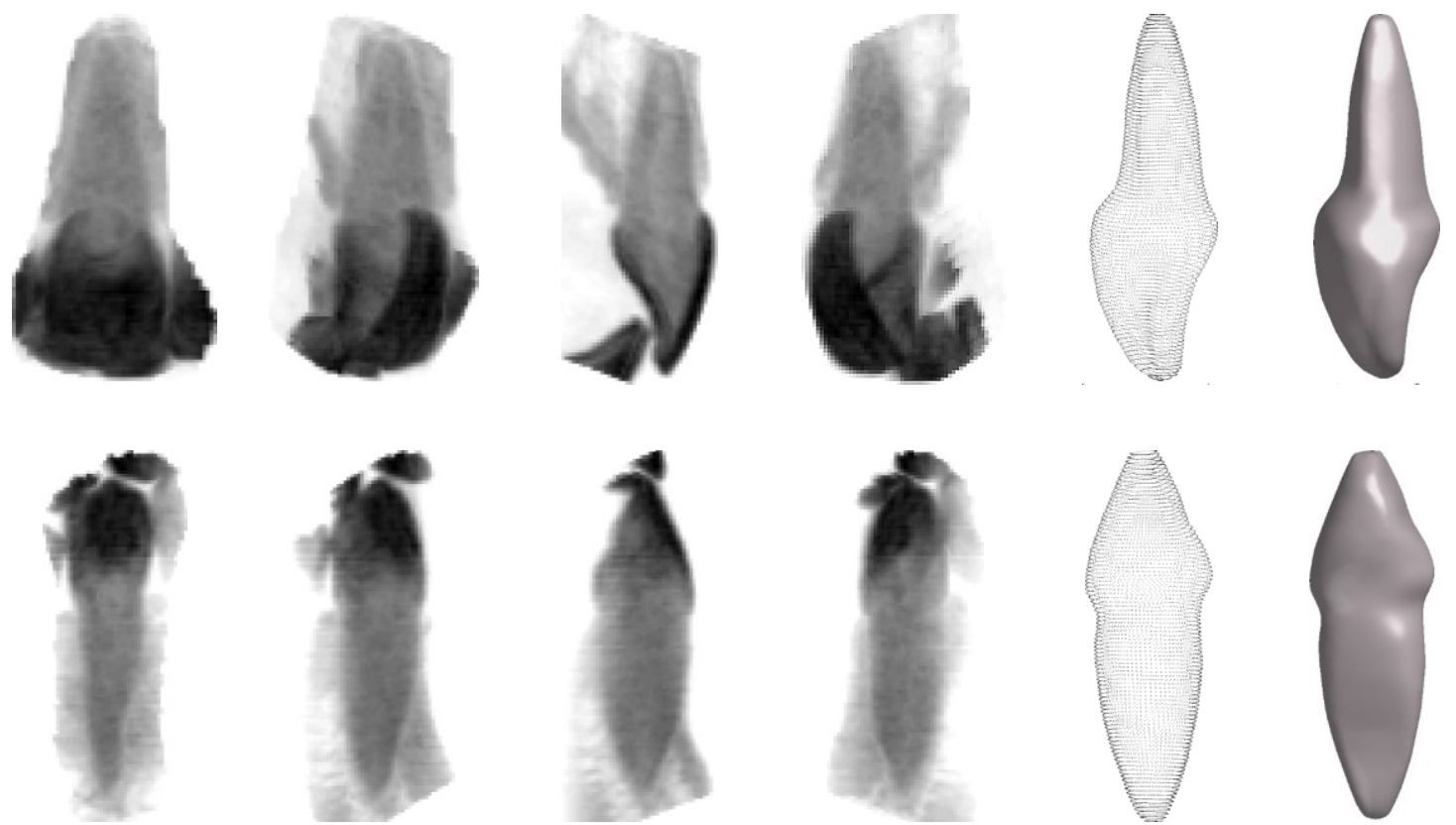

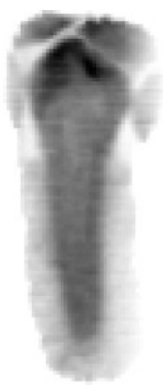

(a)

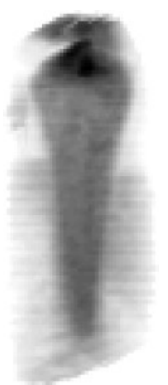

(b)

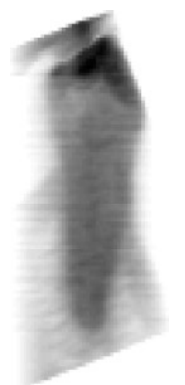

(c)

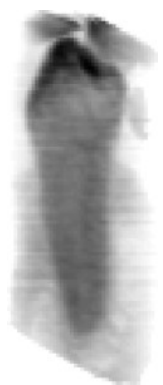

(d)

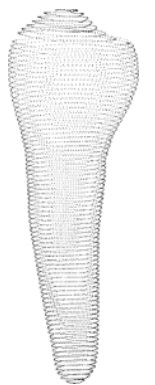

(e)

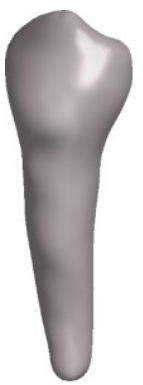

(f)

Figure 13 - Three examples of MIP images generated for a central incisor (upper), a canine (middle) and a premolar (lower) tooth (a-d); tooth point clouds obtained by spline interpolation (e); corresponding StL representations (f).

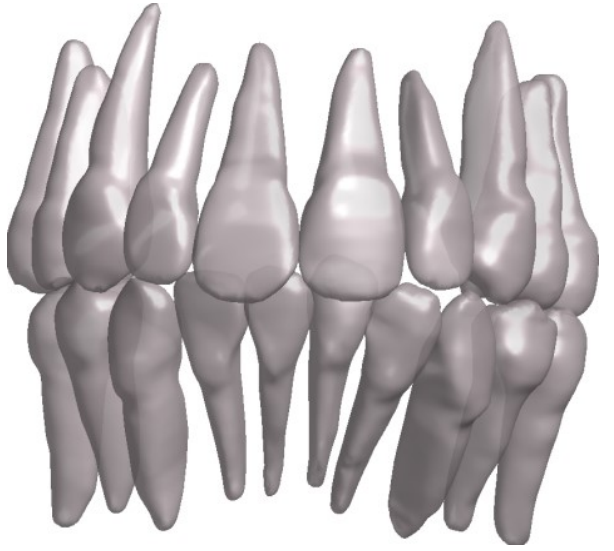

(a)

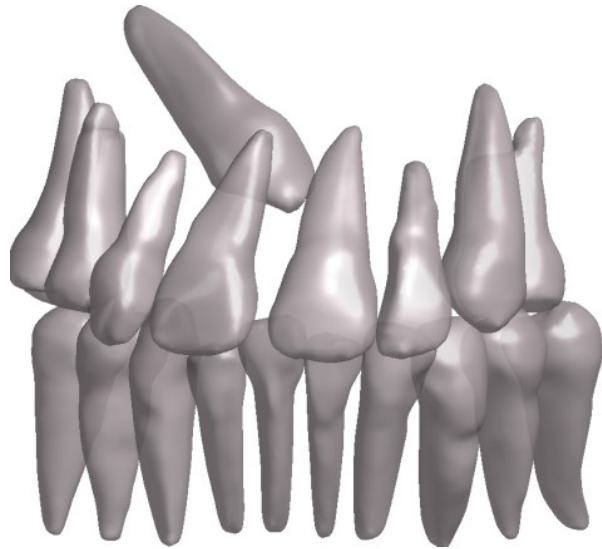

(b) 


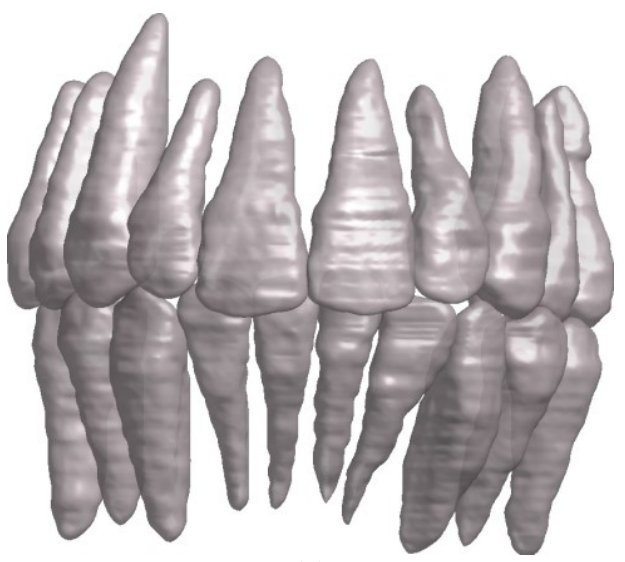

(c)

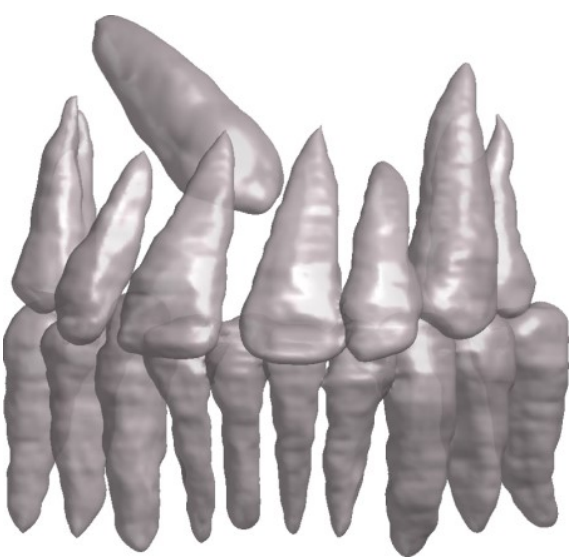

(d)

Figure 14 - Tooth geometries obtained by processing the cases described in Figure 11-a and 11-b by using the proposed approach (a) and (b), respectively, and the manual segmentation process (c) and (d), respectively.
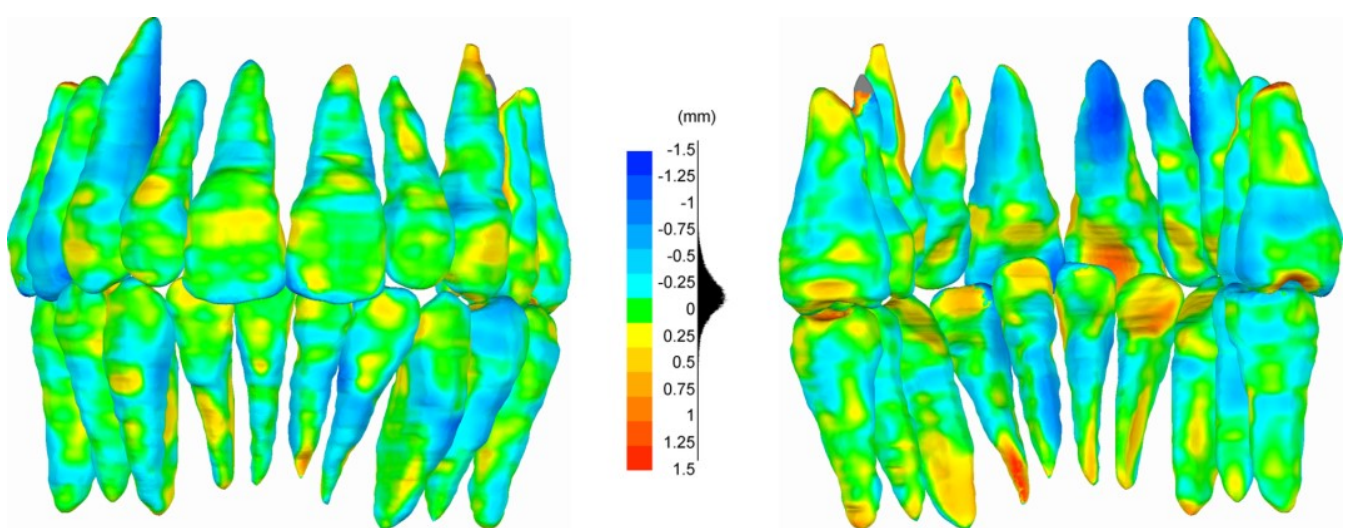

Figure 15 - Full field discrepancies between the reference model and the tooth geometries obtained by adopting the proposed approach.

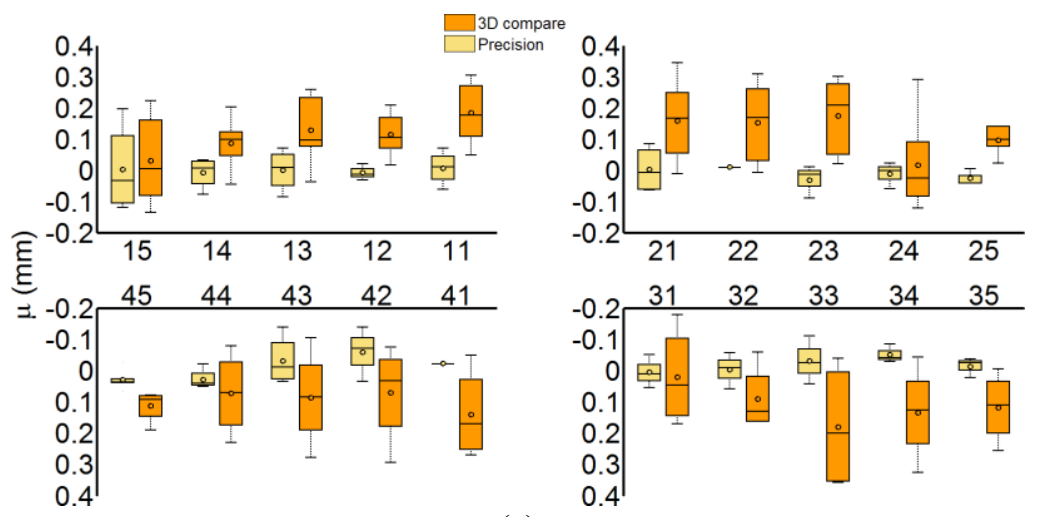

(a)

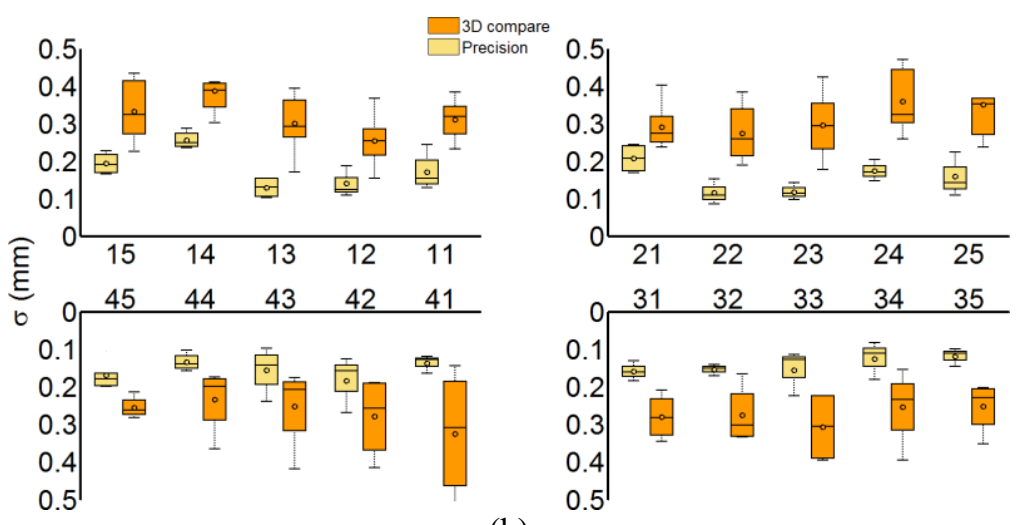

(b)

Figure 16 - Mean $\mu$ (a) and standard deviation $\sigma(\mathrm{b})$ values of the discrepancies between reconstructed teeth and reference data. 

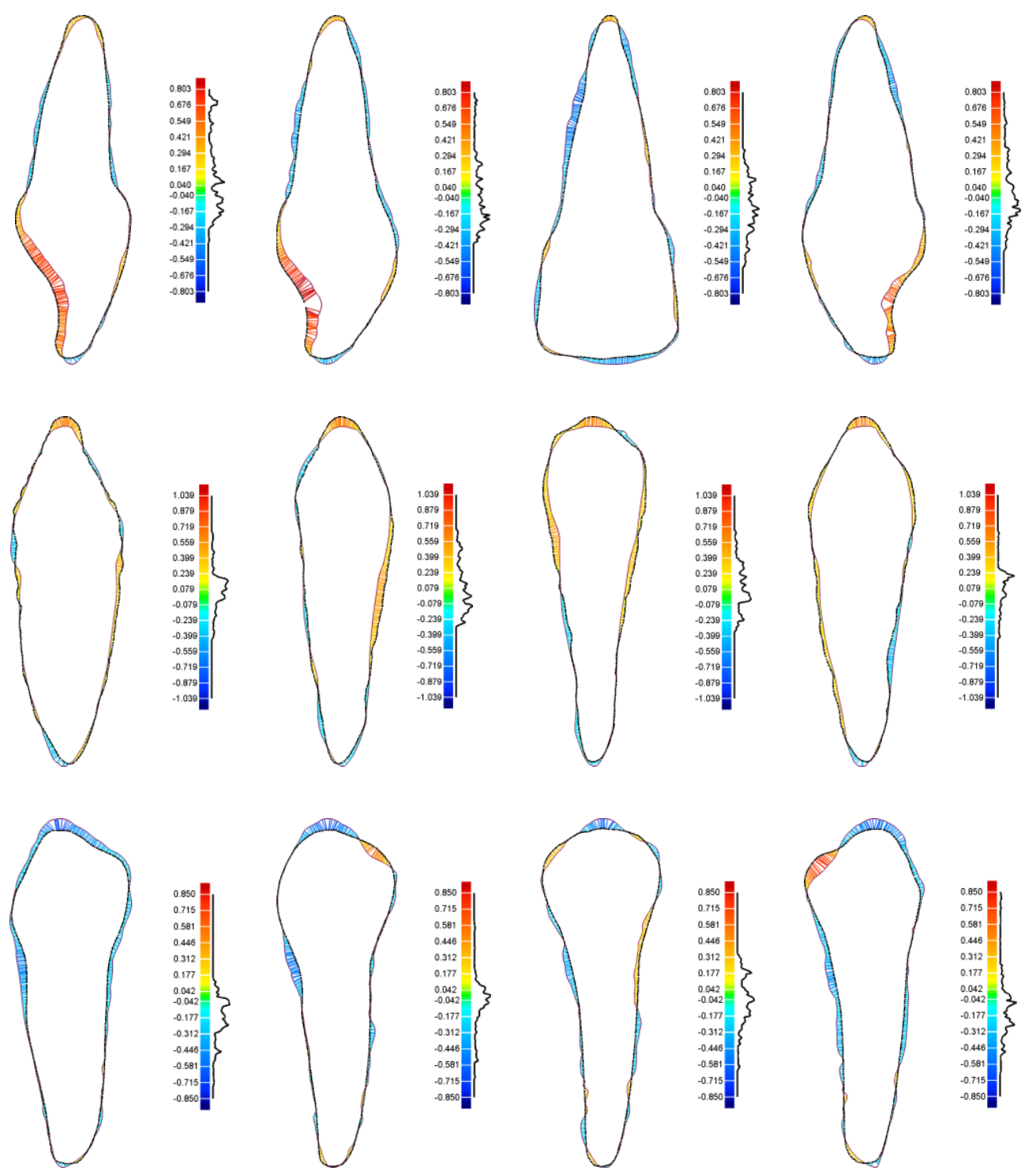

Figure 17 - 2D comparison (expressed in $\mathrm{mm}$ ) between reconstructed teeth (dashed black line) and reference data (continuous red line) identified over the four reference planar sections for teeth shown in Figure 12.

\section{Conclusions}

In the field of orthodontic treatments planning, the dental anatomy-awareness facilitates a better prediction of tooth movements, which are strictly dependent on the root geometrical attributes. In clinical practice, CBCT volumetric data are used with the aim at segmenting complete and individual tooth geometries. However, most of the existent methodologies are based on slice-by-slice segmentation processes of CBCT scans, resulting in time-consuming procedures.

In this paper, a novel approach to model tooth geometries from CBCT data has been developed. The method exploits the generation of a small set of multi-planar reformation images along four significant directions for each target tooth. The identification of these directions is driven by the 
specific anatomical geometry. The reformation images greatly enhance the clearness of the target tooth contours, which are then extracted and used to automatically model the overall 3D tooth shape through a B-spline representation. The described technique is not in contrast to other methods, rather represents an alternative methodology, which differentiates from standard approaches since it is not based on a slice-wise segmentation of standard orthogonal views. The key feature of the proposed methodology consists in requiring interactive contouring of target teeth by using a few significant images created by anatomy-driven fundamentals. The digital imaging process provides reliable geometrical data for the downstream tooth B-spline modelling. The shape modelling procedure yields geometrical approximations of individual teeth, which are spatially located with reference to the CBCT coordinate system, at the same time providing the dental arrangement. The proposed anatomydriven methodology allows dental practitioners to easily work with small sets of familiar enhanced images, rather than complex volumetric data.

Even if the approach provides tooth shape approximations, several dental treatments, such as orthodontic correction planning, would benefit of an estimation of the root geometries with the relative inertia. Clearly, the accuracy of a tooth model could be improved by increasing the number of significant viewing directions. A greater number of MIP images could be generated and processed to the detriment of computing time since the amount of user interaction is certainly greater with respect to other existing automatic approaches (i.e., level set method as in [3]). However, the structure of the presented segmentation pipeline facilitates the introduction of automatic contouring processes (i.e., based on intensity gradient analyses, snakes approach, level set approach), which could be applied on the MIP images created by the described anatomy-driven methodology. In this paper, this issue has not been considered since it would not have given any further significant contribution to the novelty of the overall approach. The described tooth segmentation methodology has been specifically designed for the reconstruction of anterior and premolar teeth, which usually present mono-radicular anatomies. Future research activities could be focused on the generalisation of the proposed approach to multi-radicular and/or multi-cusped shapes.

\section{References}

[1] Scarfe WC, Farman AG, Sukovic P. Clinical applications of cone-beam computed tomography in dental practice. Journal of the Canadian Dental Association. 2006;72:75-80.

[2] Duy NT, Lamecker H, Kainmueller D, Zachow S. Automatic detection and classification of teeth in CT data. Proceedings of the 15th international conference on Medical Image Computing and Computer-Assisted Intervention - Volume Part I. Nice, France: Springer-Verlag; 2012. p. 609-16.

[3] Ji DX, Ong SH, Foong KWC. A level-set based approach for anterior teeth segmentation in cone beam computed tomography images. Comput Biol Med. 2014;50:116-28. 
[4] Gan Y, Xia Z, Xiong J, Zhao Q, Hu Y, Zhang J. Toward accurate tooth segmentation from computed tomography images using a hybrid level set model. Medical Physics. 2015;42:14-27.

[5] Gao H, Chae O. Individual tooth segmentation from CT images using level set method with shape and intensity prior. Pattern Recogn. 2010;43:2406-17.

[6] Gao H, Chae O. Touching tooth segmentation from CT image sequences using coupled level set method. 5th International Conference on Visual Information Engineering2008. p. 382-7.

[7] Yau HT, Yang TJ, Chen YC. Tooth model reconstruction based upon data fusion for orthodontic treatment simulation. Comput Biol Med. 2014;48:8-16.

[8] Barone S, Paoli A, Razionale A. Creation of 3D Multi-Body Orthodontic Models by Using Independent Imaging Sensors. Sensors. 2013;13:2033-50.

[9] Kihara T, Tanimoto K, Michida M, Yoshimi Y, Nagasaki T, Murayama T, et al. Construction of orthodontic setup models on a computer. Am J Orthod Dentofac Orthop. 2012;141:806-13.

[10] Kretschmer J, Soza G, Tietjen C, Suehling M, Preim B, Stamminger M. ADR - Anatomy-Driven Reformation. Ieee T Vis Comput Gr. 2014;20:2496-505.

[11] Otsu N. A threshold selection method from gray-level histograms. IEEE Transactions on Systems, Man and Cybernetics. 1979;9:62-6.

[12] Tohnak S, Mehnert A, Crozier S, Mahoney M. Synthesizing panoramic radiographs by unwrapping dental CT data. Conf Proc IEEE Eng Med Biol Soc 2006;1:3329-32. 2006:1484-7.

[13] Paulo HJA, Thiago FdM, Jorge VLdS, Helio P, Rui BR. Automatic reconstruction of dental CT images using optimization. Biodental Engineering III: CRC Press; 2014. p. 57-62.

[14] Akhoondali H, Zoroofi RA, Shirani G. Fully automatic extraction of panoramic dental images from CTscan volumetric data of the head. J Applied Sci. 2009;9:2106-14.

[15] Sa-ing V, Wangkaoom K, Thongvigitmanee SS. Automatic Dental Arch Detection and Panoramic Image Synthesis from CT Images. Ieee Eng Med Bio. 2013:6099-102.

[16] Han B, Chen L, Cai Z, Pu F, Li D, Li S, et al. An automatic method of synthesizing panoramic radiograph by unwrapping dental CT image. International Conference on Mechatronic Science, Electric Engineering and Computer (MEC)2011. p. 1094-6.

[17] 3DSlicer. A multi-platform, free and open-source software package for visualization and medical image computing, http://www.slicer.org/ (accessed 19 January 2015).

[18] ISO 3950:2009. Dentistry - Designation system for teeth and areas of the oral cavity. 